\title{
Development and Validation of a Nomogram for Predicting Overall Survival in Pediatric Patients with Atypical Teratoid/ Rhabdoid Tumors
}

\author{
Yao LIU', Xiao PENG², Tingting ZENG ${ }^{2}$ \\ ${ }^{1}$ Lishui Municipal Central Hospital, Department of Orthopedics, Lishui, China \\ ${ }^{2}$ Lishui Municipal Central Hospital, Department of Neurology, Lishui, China \\ Corresponding author: Tingting ZENG zengtt2408@163.com
}

\section{ABSTRACT}

AIM: To construct a reliable prediction model for pediatric atypical teratoid/rhabdoid tumor (ATRT) patients.

MATERIAL and METHODS: Population-based data of patients diagnosed with intracranial ATRT were extracted from the National Cancer Institute's Surveillance, Epidemiology, and End Results database. These patients were randomly assigned into training and validation cohorts at a ratio of 2:1. Univariable and multivariable Cox analyses were conducted to determine independent factors of overall survival (OS). A nomogram was then developed using the covariates with the best prognostic value, and the predictive performance of the nomogram was assessed by calibration curves, concordance index, time-dependent receiver operating characteristic curve analysis, and decision curve analysis.

RESULTS: A total of 267 cases were included. The OS rates at 6 months, 1 year, and 3 years were $61.6 \%, 50.1 \%$, and $35.4 \%$, respectively. The results of multivariable Cox analysis showed that tumor extension, surgery type, radiotherapy, and chemotherapy were independent prognostic indicators. A nomogram integrating these factors was established to predict the 6-month, 1-year, and 3 -year OS rates. This prediction model was validated in the validation cohort. The nomogram had favorable predictive performance and discrimination ability.

CONCLUSION: We developed and validated a novel nomogram with favorable discrimination ability to predict prognosis for newly diagnosed pediatric ATRT patients. Although additional validation is required, this may be a useful tool in clinical decision making.

KEYWORDS: Atypical teratoid/rhabdoid tumors, SEER program, Nomograms, Predict, Overall survival

\section{INTRODUCTION}

A typical teratoid/rhabdoid tumors (ATRTs), which are characterized by multilineage differentiation and pathologically primitive phenotypes, are embryonal central nervous system (CNS) malignancies that mainly occur in children younger than 3 years old. ATRTs rarely arise in teens and adults $(3,14,16,18)$. Epidemiologically, ATRT only accounts for $1-2 \%$ of all pediatric brain tumors; however, it is the most common CNS tumor in the first year of life (18). The age-standardized incidence rate for pediatric ATRTs is approximately 1.38 per 100,000 person years, and $14-21 \%$ of cases have metastatic tumor dissemination at initial diagnosis $(2,3,25,27)$.

Despite recent advances in technologies and treatment strategies, the prognosis of ATRT is still dismal, with a reported median survival around 1 year $(2,9)$. To date, trimodality therapy consisting of surgical resection, chemotherapy, and radiotherapy is recommended. However, radiation therapy for younger children remains controversial in consideration of its CNS toxicity (8). Given the diverse histopathological features of this disease and the lack of consensus on therapeutic strategies, the outcomes across individual patients are distinct, 
rendering it quite difficult to predict survival. Therefore, tools to accurately estimate the prognosis of patients with ATRT are greatly needed and can contribute to clinical management.

Nomograms, reliable visual calculations integrating independent-related variables identified by statistical methods to predict patient survival probability, have been widely applied in oncology $(20,29-31)$. The National Cancer Institute's Surveillance, Epidemiology, and End Results (SEER) database is a valuable resource for cancer research and has been advocated for tumor study. Therefore, in this study, we used this publicly accessible database to develop and validate a nomogram for survival prediction in pediatric ATRT patients in order to aid in clinical management for this disease.

\section{MATERIAL and METHODS}

\section{Study Cohort}

The population-based data of patients diagnosed with intracranial ATRT between 2000 and 2015 within 20 SEER registries were extracted. ATRT cases among pediatric patients (younger than 20 years) were identified according to anatomic site (C71.0-C71.9) and histology (9508/3) codes. We included only cases with histologically confirmed tumors; cases identified based on death certificate or autopsy were excluded. Cases with insufficient information on surgery type or tumor extension and those without a defined follow-up time or vital status were also excluded.

\section{Variable Selection}

Patient and disease characteristics and treatment data were defined based on the SEER registry. Information regarding age at diagnosis ( $<1$ year, 1-2 years, and $\geq 3$ years), sex, race, tumor site, tumor size ( $<48 \mathrm{~mm}, \geq 48 \mathrm{~mm}$, and unknown), tumor extension, surgery type, and administration of radiotherapy and chemotherapy were also obtained. Tumor extension was classified into locoregional and distant spread according to the SEER coding guidelines. Surgery types were classified as no surgery, subtotal resection, and gross total resection (GTR). The primary outcome for our analysis was overall survival (OS).

First, we summarized characteristics of the included patients with frequencies and proportions. Then, these patients were randomly assigned into training and validation cohorts at a ratio of 2:1. Univariable and multivariable Cox proportional hazards regression analyses were conducted to determine factors significantly associated with OS in the training set. We developed a survival prediction model using the nomogram method, incorporating predictive factors with the greatest prognostic value. The nomogram was further verified in the validation cohort. In addition, we employed concordance index (C-index) and time-dependent receiver operating characteristic (ROC) curve analyses to evaluate the discrimination ability of the model. The consistency of the nomogram-predicted survival with actual patient outcomes was assessed by calibration curves. Moreover, the clinical application value of the nomogram was analyzed via decision curve analysis (DCA).

\section{Statistical Analysis}

Chi-square tests were performed appropriately to compare across random sets. OS rates were estimated using the Kaplan-Meier method. The SEER ${ }^{\star}$ Stat software was used for patient data extraction, and $\mathrm{R}$ software was employed for all statistical analyses. A p value $<0.05$ was defined as statistically significant.

\section{RESULTS}

\section{Demographic and Clinicopathological Characteristics}

From 2000 to 2015, a total of 267 patients met our inclusion criteria and were included in ourstudy cohort. The demographic and clinical characteristics of pediatric patients with ATRT are presented in Table I. The majority of patients were younger than 3 years old, with $34.1 \%$ of patients being under the age of 1 year. More than half of the patients were male $(53.2 \%)$, and $76.0 \%$ were white. Tumors most frequently occurred in the supratentorial location (41.6\%) and had locoregional spread $(75.7 \%)$. Additionally, most patients received surgery, with $41.2 \%$ of patients receiving GTR. Chemotherapy $(73.0 \%)$ was the most common treatment type, whereas only $37.8 \%$ of patients were administered radiotherapy. The OS rates at 6 months, 1 year, and 3 years were $61.6 \%, 50.1 \%$, and $35.4 \%$, respectively. The baseline characteristics were comparable between the training and validation cohorts (Table l, all p>0.05).

\section{Identification of Prognostic Factors}

As shown in Table II, univariate Cox analysis revealed that age at diagnosis, tumor extension, surgery type, radiotherapy, and chemotherapy were significantly correlated with OS (all $\mathrm{p}<0.05)$. After adjusting for potential confounding factors, the results of multivariable Cox analysis showed that tumor extension (distant vs locoregional: hazard ratio [HR], 2.23, 95\% confidence interval $[\mathrm{Cl}] 1.43-3.48, \mathrm{p}<0.001$ ), surgery type (subtotal resection vs no surgery: HR, 0.26, $p<0.001$; GTR vs no surgery: HR $0.20, p<0.001$ ), radiotherapy (yes vs no: HR 0.29, 95\% Cl 0.17-0.49, p<0.001), and chemotherapy (yes vs no: HR 0.54, 95\% Cl 0.32-0.89, $\mathrm{p}<0.001$ ) remained independent prognostic factors of OS.

\section{Nomogram Development and Performance Assessment}

We next incorporated these prognostic factors into a nomogram for individual survival rate estimation. As presented in Figure 1 , the nomogram was successfully established to predict the 6-month, 1-year, and 3-year OS rates in the training cohort. The calibration curves in both the training and validation datasets demonstrated good consistency between the observed and predicted outcomes (Figure $2 A-C$ and Figure $3 A-C$ ). Favorable discrimination performance of the nomogram was also indicated by the C-index (0.799) and ROC curve analysis, which showed areas under the curve (AUCs) at 6 months, 1 year, and 3 years of $0.89,0.85$, and 0.80 , respectively, in the training cohort (Figure 2D-F). The $\mathrm{C}$-index in the validation cohort was 0.756, and the AUCs at 6 months, 1 year, and 3 years were $0.86,0.83$, and 0.76 , respectively (Figure $3 \mathrm{D}-\mathrm{F}$ ). These validated the good predictive ability of the nomogram. We further compared the indicative prognostic power of this 
Table I: Patient Characteristics in the Study

\begin{tabular}{|c|c|c|c|c|}
\hline Parameter & Total cohort, n(\%) & Training cohort, n(\%) & Validation cohort, n(\%) & $\mathbf{p}$ \\
\hline & $267(100)$ & $178(100)$ & $89(100)$ & \\
\hline Age, year & & & & 0.959 \\
\hline$<1$ & $91(34.1)$ & $63(35.4)$ & $28(31.5)$ & \\
\hline $1-2$ & $114(42.7)$ & $73(41.0)$ & $41(46.1)$ & \\
\hline$\geq 3$ & $62(23.2)$ & $42(23.6)$ & $20(22.5)$ & \\
\hline Sex & & & & 0.965 \\
\hline Male & $142(53.2)$ & $94(52.8)$ & $48(53.9)$ & \\
\hline Female & $125(46.8)$ & $84(47.2)$ & $41(46.1)$ & \\
\hline Race & & & & 0.877 \\
\hline White & $203(76.0)$ & $137(77.0)$ & $66(74.2)$ & \\
\hline Black & $33(12.4)$ & $21(11.8)$ & $12(13.5)$ & \\
\hline Others/unknown & $31(11.6)$ & $20(11.2)$ & $11(12.4)$ & \\
\hline Tumor site & & & & 0.493 \\
\hline Supratentorial & $111(41.6)$ & $71(39.9)$ & $40(44.9)$ & \\
\hline Infratentorial & $91(34.1)$ & $65(36.5)$ & $26(29.2)$ & \\
\hline Other/brain, NOS & $65(24.3)$ & $42(23.6)$ & $23(25.8)$ & \\
\hline Tumor size & & & & 0.128 \\
\hline$<48 \mathrm{~mm}$ & $105(39.3)$ & $65(36.5)$ & $40(44.9)$ & \\
\hline$\geq 48 \mathrm{~mm}$ & $108(40.4)$ & $71(39.9)$ & $37(41.6)$ & \\
\hline Unknown & $54(20.2)$ & $42(23.6)$ & $12(13.5)$ & \\
\hline \multicolumn{5}{|l|}{ Tumor extension } \\
\hline Locoregional & $202(75.7)$ & $134(75.3)$ & $68(76.4)$ & \\
\hline Distant & $65(24.3)$ & $44(24.7)$ & 21 (23.6) & \\
\hline Surgery & & & & 0.962 \\
\hline No surgery & $21(7.9)$ & $14(7.9)$ & $7(7.9)$ & \\
\hline STR & $136(50.9)$ & $91(51.1)$ & $45(50.6)$ & \\
\hline GTR & $110(41.2)$ & $73(41.0)$ & 37 (41.6) & \\
\hline Radiotherapy & & & & 0.824 \\
\hline No & $166(62.2)$ & $112(62.9)$ & $54(60.7)$ & \\
\hline Yes & $101(37.8)$ & $66(37.1)$ & 35 (39.3) & \\
\hline Chemotherapy & & & & 0.661 \\
\hline No & $72(27.0)$ & $50(28.1)$ & $22(24.7)$ & \\
\hline Yes & $195(73.0)$ & $128(71.9)$ & 67 (75.3) & \\
\hline 6-month OS rate & $61.60 \%$ & $59.80 \%$ & $65.30 \%$ & \\
\hline 1-year OS rate & $50.10 \%$ & $47.60 \%$ & $55.20 \%$ & \\
\hline 3-year OS rate & $35.40 \%$ & $34.90 \%$ & $36.40 \%$ & \\
\hline
\end{tabular}

STR: Subtotal resection, GTR: Gross total resection, OS: Overall survival. 
Liu Y. et al: Prognosis of Pediatric Atypical Teratoid/Rhabdoid Tumors

Table II: Univariable and Multivariable Cox Analyses in the Training Cohort

\begin{tabular}{|c|c|c|c|c|}
\hline \multirow[b]{2}{*}{ Parameter } & \multicolumn{2}{|c|}{ Univariable } & \multicolumn{2}{|c|}{ Multivariable } \\
\hline & HR (95\% Cl) & $\mathbf{p}$ & HR (95\% Cl) & p \\
\hline \multicolumn{5}{|l|}{ Age, year } \\
\hline$<1$ & $1[$ Reference] & & 1[Reference] & \\
\hline $1-2$ & $0.63(0.42-0.95)$ & 0.029 & $0.93(0.59-1.47)$ & 0.744 \\
\hline$\geq 3$ & $0.42(0.24-0.71)$ & 0.001 & $0.85(0.46-1.55)$ & 0.591 \\
\hline \multicolumn{5}{|l|}{ Sex } \\
\hline Male & $1[$ Reference] & & 1 [Reference] & \\
\hline Female & $1.19(0.82-1.73)$ & 0.359 & $1.12(0.76-1.67)$ & 0.561 \\
\hline \multicolumn{5}{|l|}{ Race } \\
\hline White & $1[$ Reference] & & 1 [Reference] & \\
\hline Black & $1.57(0.93-2.66)$ & 0.092 & $1.29(0.74-2.25)$ & 0.373 \\
\hline Others/unknown & $1.09(0.60-2.01)$ & 0.774 & $0.99(0.52-1.88)$ & 0.958 \\
\hline \multicolumn{5}{|l|}{ Tumor site } \\
\hline Supratentorial & 1[Reference] & & 1 [Reference] & \\
\hline Infratentorial & $0.98(0.63-1.52)$ & 0.917 & $0.96(0.59-1.58)$ & 0.886 \\
\hline Others/brain, NOS & $1.32(0.82-2.10)$ & 0.25 & $1.30(0.80-2.12)$ & 0.295 \\
\hline \multicolumn{5}{|l|}{ Tumor size } \\
\hline$<48 \mathrm{~mm}$ & $1[$ Reference] & & 1 [Reference] & \\
\hline$\geq 48 \mathrm{~mm}$ & $1.17(0.76-1.81)$ & 0.465 & $0.74(0.46-1.20)$ & 0.224 \\
\hline Unknown & $1.13(0.68-1.86)$ & 0.643 & $0.49(0.27-0.87)$ & 0.015 \\
\hline \multicolumn{5}{|l|}{ Tumor extension } \\
\hline Locoregional & $1[$ Reference] & & 1[Reference] & \\
\hline Distant & $2.10(1.40-3.15)$ & $<0.001$ & $2.23(1.43-3.48)$ & $<0.001$ \\
\hline \multicolumn{5}{|l|}{ Surgery } \\
\hline No surgery & $1[$ Reference] & & 1 [Reference] & \\
\hline STR & $0.16(0.09-0.30)$ & $<0.001$ & $0.26(0.13-0.54)$ & $<0.001$ \\
\hline GTR & $0.13(0.07-0.25)$ & $<0.001$ & $0.20(0.09-0.42)$ & $<0.001$ \\
\hline \multicolumn{5}{|l|}{ Radiotherapy } \\
\hline No & Reference & & Reference & \\
\hline Yes & $0.25(0.16-0.40)$ & $<0.001$ & $0.29(0.17-0.49)$ & $<0.001$ \\
\hline \multicolumn{5}{|l|}{ Chemotherapy } \\
\hline No & 1 [Reference] & & 1 [Reference] & \\
\hline Yes & $0.30(0.20-0.45)$ & $<0.001$ & $0.54(0.32-0.89)$ & 0.017 \\
\hline
\end{tabular}

STR: Subtotal resection, GTR: Gross total resection, OS: Overall survival. 


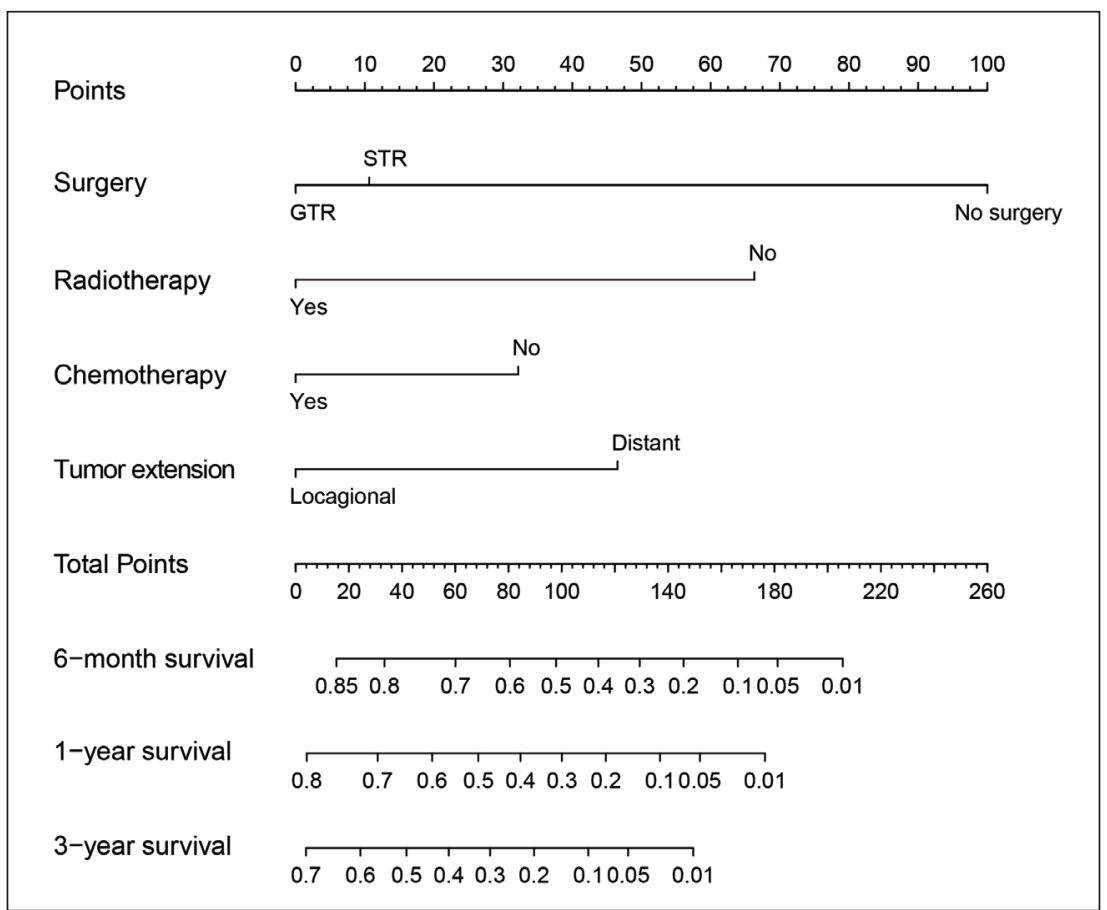

Figure 1: The novel-built nomogram predicting the 6-month, 1-year and 3-year overall survival for pediatric ATRT patients. STR: Subtotal resection, GTR: Gross total resection.

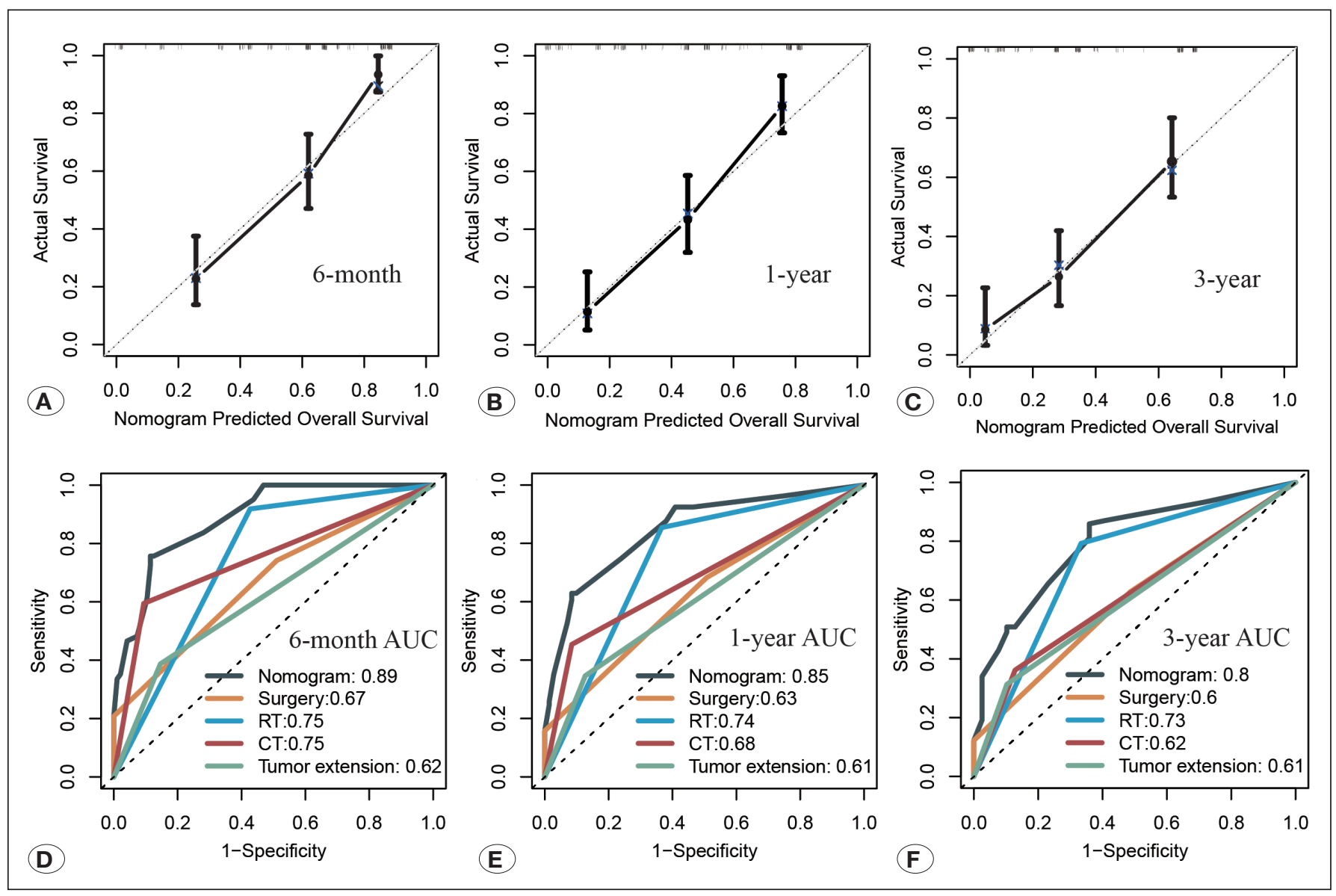

Figure 2: Calibration curves and ROC curves of the nomogram in the training cohort. Calibration plots describing A) 6-month, B) 1-year and C) 3-year OS; ROC curves predicting D) 6-month, E) 1-year and F) 3-year OS. ROC: Receiver operating characteristic, OS: Overall survival. 


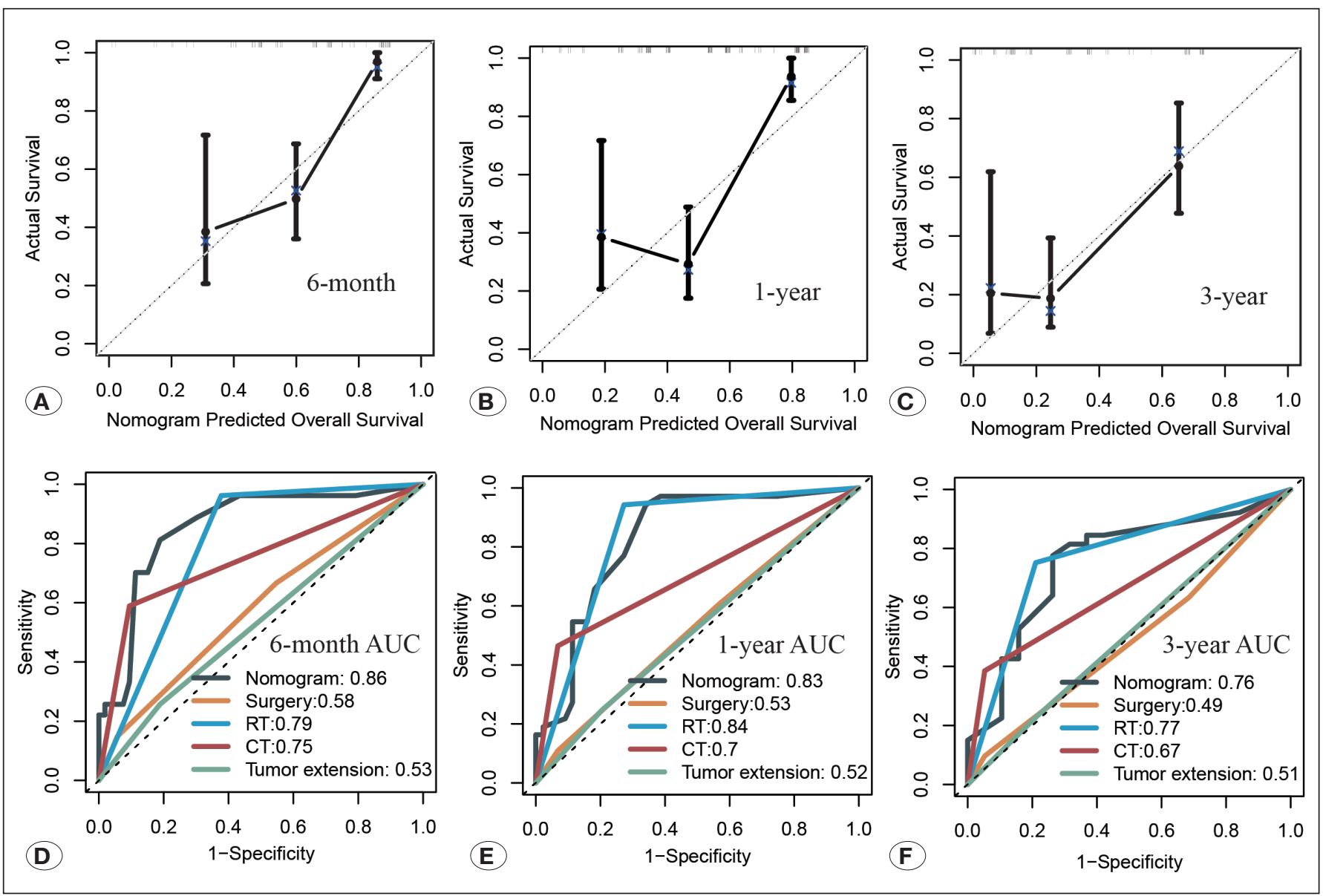

Figure 3: Calibration curves and ROC curves of the nomogram in the validation cohort. Calibration plots describing A) 6-month, B) 1-year and C) 3-year OS; ROC curves predicting D) 6-month, E) 1-year and F) 3-year OS. ROC: Receiver operating characteristic; OS: Overall survival.

novel prediction model with other scoring systems for ATRT, and we found that the nomogram had the best performance with the highest AUC. Finally, DCA showed that our model was of great clinical value in both the training and validation groups (Figure 4A-F).

\section{DISCUSSION}

The importance of survival prediction is increasingly recognized in current clinical practice. Owing to the relative rarity of ATRT, there is, to our knowledge, no prognostic prediction model for these patients. Here, we used the SEER database, which provided a pediatric ATRT cohort with a relatively large sample size, to build a nomogram for OS estimation, thereby aiding in individualized management decision-making. We also verified the greater predictive performance of the model by comparing it with traditional scoring systems for ATRT in both the training and validation cohorts. Our nomogram had the highest AUC, indicating its better predictive ability. The clinical utility of the predictive model was further proved by DCA.

In our investigation, four factors, tumor extension, surgery type, radiotherapy, and chemotherapy, were identified as independent prognostic indicators and were integrated into our prediction model. Similarly, Yamasaki et al., in a study including 38 ATRT patients, found that metastasis stage and GTR were prognostic factors for progression-free survival, whereas high-dose chemotherapy was significantly related to OS (28). However, no benefit of postoperative radiotherapy was observed in their cohort, which might be attributable to selection bias and the small sample size of their study (20). Several studies have also indicated that younger age, distant metastasis, and delayed initiation of radiotherapy have a negative impact on OS $(9,12,19,26)$. Additionally, supratentorial tumor location tends to lead to a better outcome (2). Nevertheless, in our large study, patient age and tumor location had only marginal effects on survival. In order to simplify our model for ease of use, these two factors were excluded from our nomogram.

Despite the high heterogeneity in tumor location, treatment response, and disease stage in ATRT patients, ATRTs also almost universally exhibit loss of SMARCB1, which may contribute to clinical heterogeneity (12). Recent transcription and methylation profiling studies also suggest the existence of molecular subgroups among ATRTs (11). This molecular heterogeneity should be further explored to aid in developing novel therapeutic strategies for ATRT patients. 


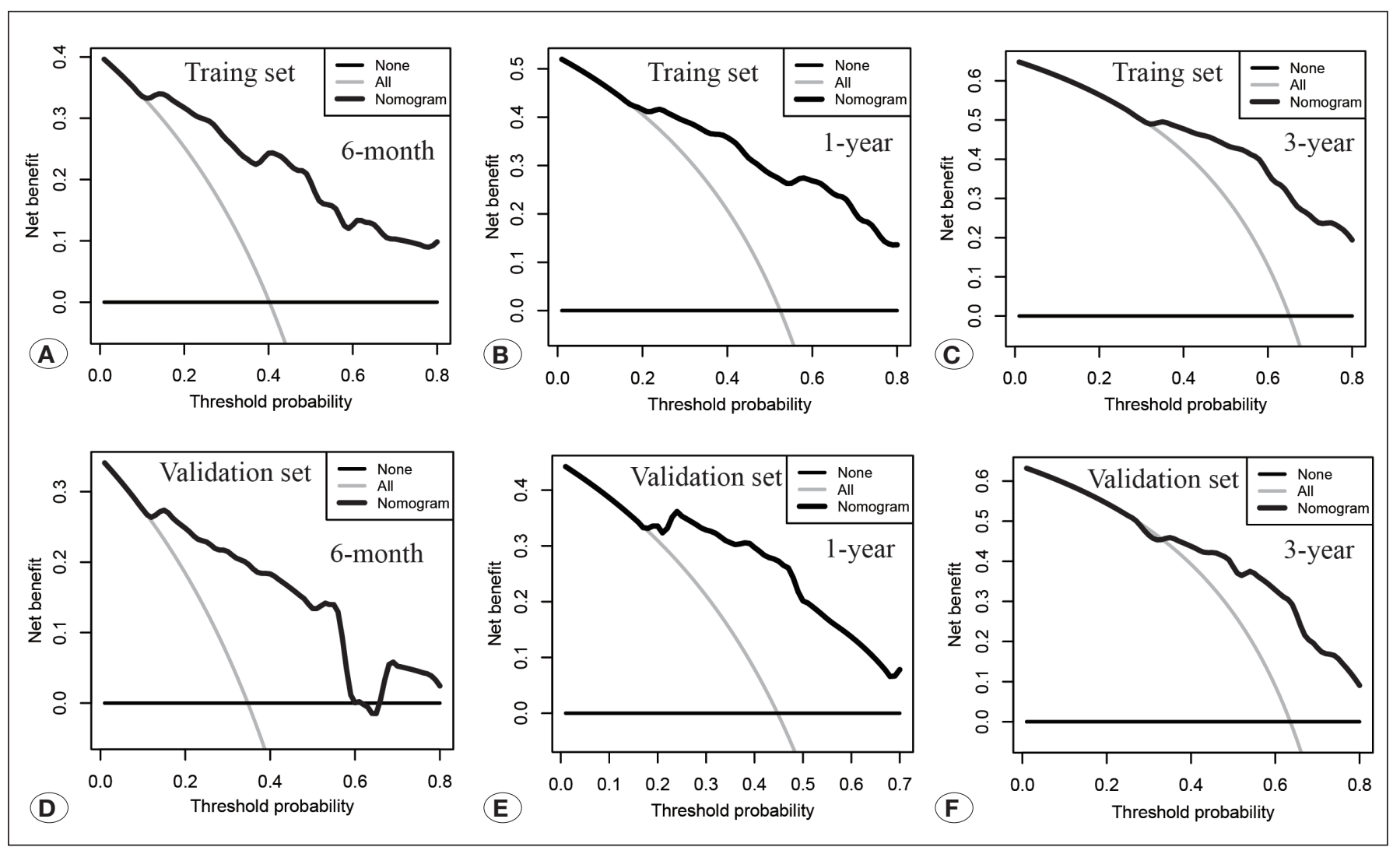

Figure 4: DCA curves of the nomogram for A) 6-month, B) 1-year and C) 3-year OS in training cohort and for D) 6-month, E) 1-year and F) 3-year OS in validation cohorts. DCA: Decision curve analysis; OS: Overall survival.

To date, the role of radiotherapy in pediatric patients with ATRT remains controversial, especially for children younger than 3 years old, because the toxicity of radiation therapy to the developing CNS can lead to long-term sequelae. Nevertheless, a number of studies have demonstrated a clinical benefit of radiotherapy with regard to survival $(1,4,5,7,10,13,15,17,22,24,25)$. Recently, Quinn et al. conducted a propensity score-matched analysis to robustly evaluate the role of radiotherapy in a cohort of 190 pediatric ATRT patients. They found that trimodality therapy could achieve better survival than surgery and chemotherapy without radiotherapy, and the benefit of radiotherapy was more significant in infants and toddlers (22). Park et al. found in their multicenter study that high-dose chemotherapy and adjuvant radiotherapy remained significant prognostic factors for progression-free survival in ATRT patients younger than 3 years old (21). Recently, the first ATRT-specific cooperative group trial, ACNS0333, was designed to evaluate the efficacy and safety of intensive postoperative chemotherapy and focal radiation for treatment of ATRTs. The results showed that intensive multimodal therapy could significantly improve outcomes for ATRT patients (23). Another prospective multiinstitutional trial also indicated that an intensive multimodality regimen could prolong both progression-free survival and OS in these patients (6). Similarly, our study also demonstrated improvement in survival with radiotherapy in the multivariable
Cox analysis. Further randomized controlled trials are required to verify the effect of radiotherapy in pediatric ATRT patients younger than 3 years.

Some limitations should be noted. First, although the SEER database can provide a large study cohort, it is dependent on accurate data coding and reporting, which might cause some bias. Second, information on target volumes, dose, and fractionation are unavailable for patients treated with radiotherapy, and detailed regimens of chemotherapy are also unknown. In addition, with great advances in molecular biology, a number of genomic markers associated with ATRT occurrence and progression have been identified; however, because the SEER database fails to provide genomic data, we were unable to analyze these factors and integrate them into our model. Finally, our prediction model needs to be evaluated carefully in other cohorts.

\section{CONCLUSION}

In our study, a novel nomogram with favorable discrimination ability was successfully constructed and validated to predict the 6-month, 1-year, and 3-year survival probabilities for newly diagnosed pediatric ATRT patients. This nomogram can promote doctor-patient communication and assist in clinical decision-making. This model could also be used to estimate significant prognostic factors in ATRTs. 


\section{REFERENCES}

1. Bishop AJ, McDonald MW, Chang AL, Esiashvili N: Infant brain tumors: Incidence, survival, and the role of radiation based on Surveillance, Epidemiology, and End Results (SEER) Data. Int J Radiat Oncol Biol Phys 82:341-347, 2019

2. Biswas A, Kashyap L, Kakkar A, Sarkar C, Julka PK: Atypical teratoid/rhabdoid tumors: Challenges and search for solutions. Cancer Manag Res 8:115-125, 2016

3. Buscariollo DL, Park HS, Roberts KB, Yu JB: Survival outcomes in atypical teratoid rhabdoid tumor for patients undergoing radiotherapy in a surveillance, epidemiology, and end results analysis. Cancer 118:4212-4219, 2012

4. Chan V, Marro A, Findlay JM, Schmitt LM, Das S: A systematic review of atypical teratoid rhabdoid tumor in adults. Front Oncol 8:567, 2018

5. Chen YW, Wong TT, Ho DM, Huang PI, Chang KP, Shiau CY, Yen $\mathrm{SH}$ : Impact of radiotherapy for pediatric CNS atypical teratoid/rhabdoid tumor (single institute experience). Int $\mathrm{J}$ Radiat Oncol Biol Phys 64:1038-1043, 2006

6. Chi SN, Zimmerman MA, Yao X, Cohen KJ, Burger P, Biegel JA, Rorke-Adams LB, Fisher MJ, Janss A, Mazewski C, Goldman S, Manley PE, Bowers DC, Bendel A, Rubin J, Turner CD, Marcus KJ, Goumnerova L, Ullrich NJ, Kieran MW: Intensive multimodality treatment for children with newly diagnosed CNS atypical teratoid rhabdoid tumor. J Clin Oncol 27(3):385-389, 2009

7. De Amorim Bernstein K, Sethi R, Trofimov A, Zeng C, Fullerton B, Yeap BY, Ebb D, Tarbell NJ, Yock TI, MacDonald SM: Early clinical outcomes using proton radiation for children with central nervous system atypical teratoid rhabdoid tumors. Int J Radiat Oncol Biol Phys 86:114-120, 2013

8. Duffner PK, Cohen ME, Thomas PR, Lansky SB: The longterm effects of cranial irradiation on the central nervous system. Cancer 56:1841-1846, 1985

9. Dufour C, Beaugrand A, Le Deley MC, Bourdeaut F, André N, Leblond P, Bertozzi Al, Frappaz D, Rialland X, Fouyssac F, Edan C, Grill J, Quidot M, Varlet P: Clinicopathologic prognostic factors in childhood atypical teratoid and rhabdoid tumor of the central nervous system: A multicenter study. Cancer 118:3812-3821, 2012

10. Elsayad K, Kriz J, Samhouri L, Haverkamp U, Straeter R, Stummer W, Eich HT: Long-term survival following additive radiotherapy in patients with atypical teratoid rhabdoid tumors. Strahlenther Onkol 192:569-581, 2016

11. Frühwald $\mathrm{MC}$, Biegel JA, Bourdeaut F, Roberts $\mathrm{CW}$, Chi SN: Atypical teratoid/rhabdoid tumors-current concepts, advances in biology, and potential future therapies. Neuro Oncol 18(6):764-778, 2016

12. Hoffman LM, Richardson EA, Ho B, Margol A, Reddy A, LafayCousin L, Chi S, Slavc I, Judkins A, Hasselblatt M, Bourdeaut F, Frühwald MC, Vibhakar R, Bouffet E, Huang A: Advancing biology-based therapeutic approaches for atypical teratoid rhabdoid tumors. Neuro Oncol 22(7):944-954, 2020

13. Lee J, Kim DS, Han JW, Suh CO: Atypical teratoid/rhabdoid tumors in children treated with multimodal therapies: The necessity of upfront radiotherapy after surgery. Pediatr Blood Cancer, 2017 (Online ahead of print)
14. Louis DN, Perry A, Reifenberger G, von Deimling A, FigarellaBranger D, Cavenee WK, Ohgaki H, Wiestler OD, Kleihues P, Ellison DW: The 2016 World Health Organization Classification of tumors of the central nervous system: A summary. Acta Neuropathol 131:803-820, 2016

15. McGovern SL, Okcu MF, Munsell MF, Kumbalasseriyil N, Grosshans DR, McAleer MF, Chintagumpala M, Khatua S, Mahajan A: Outcomes and acute toxicities of proton therapy for pediatric atypical teratoid/rhabdoid tumor of the central nervous system. Int J Radiat Oncol Biol Phys 90:1143-1152, 2014

16. Nesvick CL, Lafay-Cousin L, Raghunathan A, Bouffet E, Huang AA, Daniels DJ: Atypical teratoid rhabdoid tumor: Molecular insights and translation to novel therapeutics. J Neurooncol 150(1):47-56, 2020

17. Nesvick CL, Nageswara Rao AA, Raghunathan A, Biegel JA, Daniels DJ: Case-based review: Atypical teratoid/rhabdoid tumor. Neurooncol Pract 6(3):163-178, 2019

18. Ostrom QT, Chen Y, P MdB, Ondracek A, Farah P, Gittleman H, Wolinsky Y, Kruchko C, Cohen ML, Brat DJ, BarnholtzSloan JS: The descriptive epidemiology of atypical teratoid/ rhabdoid tumors in the United States, 2001-2010. Neuro Oncol 16:1392-1399, 2014

19. Pai Panandiker AS, Merchant TE, Beltran C, Wu S, Sharma S, Boop FA, Jenkins JJ, Helton KJ, Wright KD, Broniscer A, Kun LE, Gajjar A: Sequencing of local therapy affects the pattern of treatment failure and survival in children with atypical teratoid rhabdoid tumors of the central nervous system. Int $\mathrm{J}$ Radiat Oncol Biol Phys 82:1756-1763, 2012

20. Pan Z, You H, Bu Q, Feng X, Zhao F, Li Y, Lyu J: Development and validation of a nomogram for predicting cancer-specific survival in patients with Wilms' tumor. J Cancer 10:52995305, 2019

21. Park M, Han JW, Hahn SM, Lee JA, Kim JY, Shin SH, Kim DS, Yoon HI, Hong KT, Choi JY, Kang HJ, Shin HY, Phi JH, Kim SK, Lee JW, Yoo KH, Sung KW, Koo HH, Lim DH, Shin HJ, Kim H, Koh KN, Im HJ, Ahn SD, Ra YS, Baek HJ, Kook H, Jung TY, Choi HS, Kim CY, Park HJ, Lyu CJ: Atypical teratoid/rhabdoid tumor of the central nervous system in children under the age of 3 years. Cancer Res Treat 53(2):378-388, 2021

22. Quinn TJ, Almahariq MF, Siddiqui ZA, Thompson AB, Hamstra DA, Kabolizadeh P, Gowans KL, Chen PY: Trimodality therapy for atypical teratoid/rhabdoid tumor is associated with improved overall survival: A surveillance, epidemiology, and end results analysis. Pediatr Blood Cancer 66:e27969, 2019

23. Reddy AT, Strother DR, Judkins AR, Burger PC, Pollack IF, Krailo MD, Buxton AB, Williams-Hughes C, Fouladi $M$, Mahajan A, Merchant TE, Ho B, Mazewski CM, Lewis VA, Gajjar A, Vezina LG, Booth TN, Parsons KW, Poss VL, Zhou T, Biegel JA, Huang A: Efficacy of high-dose chemotherapy and three-dimensional conformal radiation for atypical teratoid/ rhabdoid tumor: A report from the children's oncology group trial ACNS0333. J Clin Oncol 38(11):1175-1185, 2020

24. Squire SE, Chan MD, Marcus KJ: Atypical teratoid/rhabdoid tumor: the controversy behind radiation therapy. J Neurooncol 81(1):97-111, 2007 
25. Tekautz TM, Fuller CE, Blaney S, Fouladi M, Broniscer A, Merchant TE, Krasin M, Dalton J, Hale G, Kun LE, Wallace D, Gilbertson RJ, Gajjar A: Atypical teratoid/rhabdoid tumors (ATRT): Improved survival in children 3 years of age and older with radiation therapy and high-dose alkylator-based chemotherapy. J Clin Oncol 23:1491-1499, 2005

26. von Hoff K, Hinkes B, Dannenmann-Stern E, von Bueren AO, Warmuth-Metz M, Soerensen N, Emser A, Zwiener I, Schlegel PG, Kuehl J, Frühwald MC, Kortmann RD, Pietsch T, Rutkowski S: Frequency, risk-factors and survival of children with atypical teratoid rhabdoid tumors (AT/RT) of the CNS diagnosed between 1988 and 2004, and registered to the German HIT database. Pediatr Blood Cancer 57:978-985, 2011

27. Woehrer A, Slavc I, Waldhoer T, Heinzl H, Zielonke N, Czech T, Benesch M, Hainfellner JA, Haberler C: Incidence of atypical teratoid/rhabdoid tumors in children: A population-based study by the Austrian Brain Tumor Registry, 1996-2006. Cancer 116:5725-5732, 2010
28. Yamasaki K, Kiyotani C, Terashima K, Watanabe Y, Kanamori M, Koga Y, Hata N, Iwasaki F, Goto H, Koh K, Kurihara J, Tokunaga S, Arakawa Y, Hasegawa D, Kosaka Y, Hara J: Clinical characteristics, treatment, and survival outcome in pediatric patients with atypical teratoid/rhabdoid tumors: A retrospective study by the Japan Children's Cancer Group. J Neurosurg Pediatr 2019 (Online ahead of print)

29. Yu C, Zhang Y: Development and validation of prognostic nomogram for young patients with gastric cancer. Ann Transl Med 7:641, 2019

30. Zhang H, Ma G, Du S, Sun J, Zhang Q, Yuan B, Luo $X$ : Nomogram for predicting cancer specific survival in inflammatory breast carcinoma: A SEER population-based study. Peer J 7:e7659, 2019

31. Zheng W, Huang $Y$, Chen $H$, Wang $N$, Xiao W, Liang $Y$, Jiang $X$, Su W, Wen S: Nomogram application to predict overall and cancer-specific survival in osteosarcoma. Cancer Manag Res 10:5439-5450, 2018 